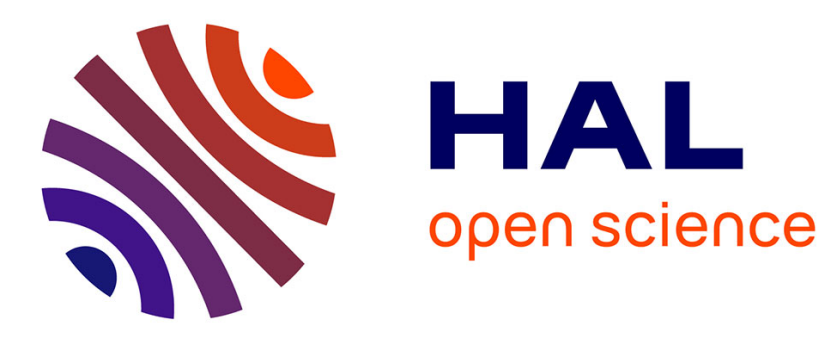

\title{
RECONSTRUCTION OF VERTICAL DISTRIBUTIONS OF SOUND AND FLOW VELOCITIES AT STRONG OCEANIC CURRENTS VIA INVERSION OF ACOUSTIC TRAVEL TIMES
}

O. Godin, D. Mikhin, S. Molchanov

\section{To cite this version:}

O. Godin, D. Mikhin, S. Molchanov. RECONSTRUCTION OF VERTICAL DISTRIBUTIONS OF SOUND AND FLOW VELOCITIES AT STRONG OCEANIC CURRENTS VIA INVERSION OF ACOUSTIC TRAVEL TIMES. Journal de Physique IV Proceedings, 1992, 02 (C1), pp.C1-953-C1-956. 10.1051/jp4:19921208 . jpa-00251174

HAL Id: jpa-00251174 https://hal.science/jpa-00251174

Submitted on 1 Jan 1992

HAL is a multi-disciplinary open access archive for the deposit and dissemination of scientific research documents, whether they are published or not. The documents may come from teaching and research institutions in France or abroad, or from public or private research centers.
L'archive ouverte pluridisciplinaire HAL, est destinée au dépôt et à la diffusion de documents scientifiques de niveau recherche, publiés ou non, émanant des établissements d'enseignement et de recherche français ou étrangers, des laboratoires publics ou privés. 


\title{
RECONSTRUCTION OF VERTICAL DISTRIBUTIONS OF SOUND AND FLOW VELOCITIES AT STRONG OCEANIC CURRENTS VIA INVERSION OF ACOUSTIC TRAVEL TIMES
}

\author{
O.A. GODIN, D.Yu. MIKHIN and S.Ya. MOLCHANOV \\ Krasikova 23, P.P. Shirshov Institute of Oceanology, the USSR Academy of Sciences, 117218 \\ Moscow, USSR
}

\begin{abstract}
Effect of strong ocean currents on sound propagation is simulated numerically in framework of ray acoustics. For acoustic remote sensing of sound speed and flow velocity a nonperturbative approach is proposed and implemented. Under environmental conditions typical to the Gulf Stream applicability of this approach is demonstrated.
\end{abstract}

\begin{abstract}
L'influence du courant oceanique fort a la propagation du son est reproduite numeriquement par l'approximation de l'acoustique rayonnante. Pour le sentiment acoustique loign de la vitesse sonoree et de la rapidit du flot l'approche nonperturbatrice est propos et realisee. Sous les conditionis de l'environnement typiquees pour Gulf Stream l'applicabilit de cette approche est demonstree.
\end{abstract}

Tomographic mapping of ocean currents seems to be one of the most promising tools to study dynamics of the ocean [1-3]. This paper is a part of theoretical study of possibilities of acoustic monitoring of powerful oceanic flows from moving ships. Effect of strong current such as the Gulf stream on acoustic signals propagating in opposite directions is estimated by numerical simulation in the framework of ray acoustic. Reconstruction of vertical distributions of sound and horizontal flow velocities by nonperturbative inversion of acoustic travel times is considered. Some properties of this solution are studied using simulated data. Environmental conditions close to that of the Gulf Stream are implied in all these calculations.

The influence of currents on the propagation of acoustic signals depends upon many factors and first of all upon the flow velocity $\vec{u}$, which is typical for medium in hand. The most pronounced azimuth variations of acoustic fields appear to take place in the regions of powerful flows, like the Gulf Stream or the Kuroshio. Such a currents have sufficiently high flow velocity at the surface and intervening the ocean up to a large depth. To simulate all the effects induced by ocean currents one should certainly use computer code which makes it possible to calculate acoustic field in range-dependent moving media. However, if the source-receiver separation is not too large, adequate estimates can be obtained in the framework of the layered model of the ocean.

Departing from explicit integral expressions for eikonal, pressure amplitude at a ray and for its trajectory, an efficient computer code was developed by the authors to simulate high-frequency acoustic field in stratified moving media [4]. This code was applied to study a shift of convergence zone's boundaries induced by currents as well as differences in transmission loss and acoustic travel times in reciprocal transmissioll. For more details the reader is referred to [4].

The powerful currents turns out to exert an evident influence on the parameters of acoustic signals in the deep ocean, which is large enough to be measured using the modern experimental equipment. The vertical displacement of shadow zone's boundary 
modern experimental equipment. The vertical displacement of shadow zone's boundary at fixed distance from the source mounts to $100-150$ meters. The relative changes in the horizontal distance $D$ from source to the beginning of first convergence zone approximately equals $\Delta \mathrm{D} / \mathrm{D} \cong|\Delta \overrightarrow{\mathrm{u}}| /|\Delta \mathrm{c}|$, where $\Delta \overrightarrow{\mathrm{u}}$ and $\Delta \mathrm{c}$ are variations of sound and $\mathrm{flow}$ velocities respectively. For typical values $|\Delta \mathrm{c}|=50 \mathrm{~m} / \mathrm{s},|\overrightarrow{\mathrm{u}}|=1.5 \mathrm{~m} / \mathrm{s}$ and $\mathrm{D} \cong 50 \mathrm{~km}$ in accordance with this estimate the shift of convergence zone proved to be $\Delta \mathrm{D}=1.5-2 \mathrm{~km}$. Due to this reason considerably acoustic reciprocity breaking may occur in the vicinity of shadow zone's boundaries. The difference in transmission loss with and against the current exceeds $15 \mathrm{~dB}$ in the frequency domain about $500 \mathrm{~Hz}$. In the reciprocal tomography experiments data set consists of differential travel times $\Delta T$. The flow velocity usually reaches its maximum values near the ocean surface. That's why the shadow turning rays have greater magnitudes of $\Delta \mathrm{T}$ about $15-20 \mathrm{~ms}$ per cycle of ray.

Some of these results are illustrated by graphs of sound fleld intensifies versus depth (Fig.1), calculated for signals propagating downstream (solid curve) and upstream (dashed curve). Profiles of sound speed $c$ and flow velocity $u$, used in this simulation, are shown at Fig.1a. They correspond to CTD measurements during the survey of the Gulf Stream meander [5]. The point monopole source with frequency $f=500$ $\mathrm{Hz}$ is situated at depth $\mathrm{z}_{\mathrm{s}}=500 \mathrm{~m}$. The horizontal separation from the source equals

$\mathrm{R}=58 \mathrm{~km}$. The absorption of sound was not taking into account. Surface and bottom assumed to be flat and perfectly reflecting. In this example the shadow zone's boundary passes at depth $z_{p}=290 \mathrm{~m}$ and $z_{m}=220 \mathrm{~m}$ for rays, propagating with and against the current. It results in large differences in acoustic intensity between these horizons, which mounts to $15 \mathrm{~dB}$. These deviations are clearly observable in spite of presence of bottom reflected and surface reflected rays.

To simulate propagation of acoustic signals in moving ocean some approximate methods are widely used, which are based on substitution of real medium by a stationary one with some effective sound speed (ESS) depending on flow velocity [69]. Conditions of validity of such approximation was addressed in paper [4] using analytical comparison of exact and approximate solutions as well as numerical simulation. ESS was taken to be $c_{e}=c+\hat{u}$, where $\hat{u}$ is the projection of $\vec{u}$ on the vertical plane containing both source and receiver. It is shown that under conditions of guided propagation the main error in transmission loss is caused by errors in phases of separate eigenrays $\delta \Phi=2 \pi f R O\left(|\vec{u}| x^{2} / c^{2}\right)$, accumulating with distance. Here $R$ is source-receiver separation, $f$ is the signal frequency and $\chi$ is the grazing angle of the ray involved. Bar denotes the average value along the ray path. The error grows with frequency $f$ and distance $R$. This conclusion is in a good agreement with computer simulations. Let us consider Fig. 2 for example. The vertical dependence of acoustic field intensity at the distance $R=280 \mathrm{~km}$ downstream was calculated with $f=1$ $\mathrm{kHz}$ (Fig. $\mathrm{Za}$ ) and $f=5 \mathrm{kHz}$ (Fig. 2b), Other conditions being equal to those of Fig. 1 . Solid curve and squares represent the acoustic pressure amplitude obtained via exact formulas of ray acoustic of moving media and within ESS approximation respectively. Discrepancies between these two graphs rises rapidly with frequency increase.

Determining sound speed $c$ and current velocity $\vec{u}$ by means of ocean acoustic tomography implies solution of an inverse problem. This paper treats a kinematic inverse problem (KIP), where acoustic travel times $T$ for various source-receiver sepnrations are considered as input data.

If currents are slow, the inverse problem may be addressed via linear inversion [2]. However, linearization with respect to $\vec{u}$ isn't usually permissible, when sound propagates through powerful currents such as the Gulf Stream. Another approach, which was d'veloped in paper [10], consists in generalizations of some results, obtained

earlier using Abel's transformation for media at rest, on moving media. The main advantage of our methods is their nonperturbative nature. That is, any reference profiles of $r$ and $\vec{u}$ are not used during the inversion.

The inversion formulas mentioned above involve continuous data. It is of great importance for practical implementation, what amount of input information is really needed to find $c(z)$ and $\vec{u}(z)$. This question is addressed at Fig.3. Original profiles 


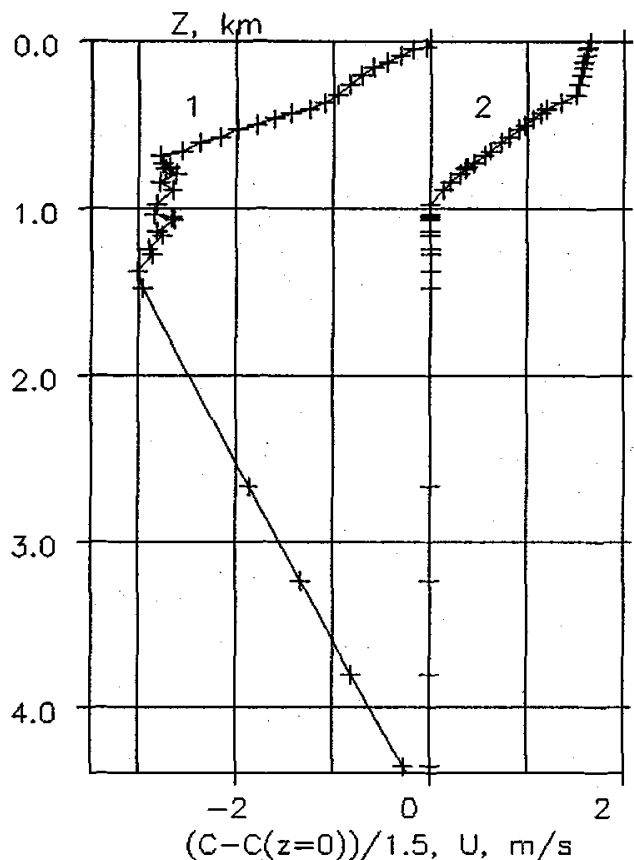

a)

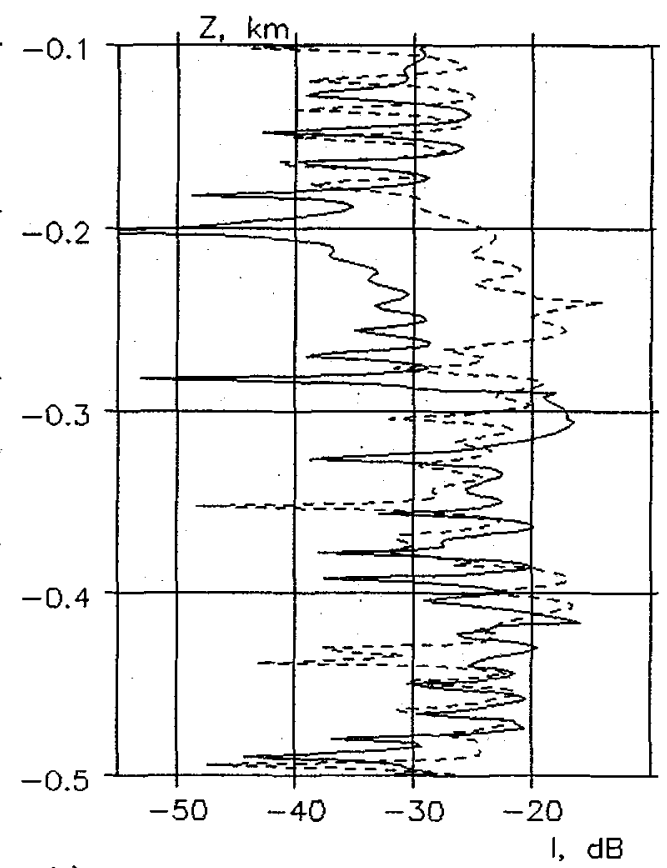

b)

Fig.1. (a) The sound speed (1) and the flow velocity (2) vertical profiles. (b) Transmission loss vs depth at the distance $R=58 \mathrm{~km}$ from the source.

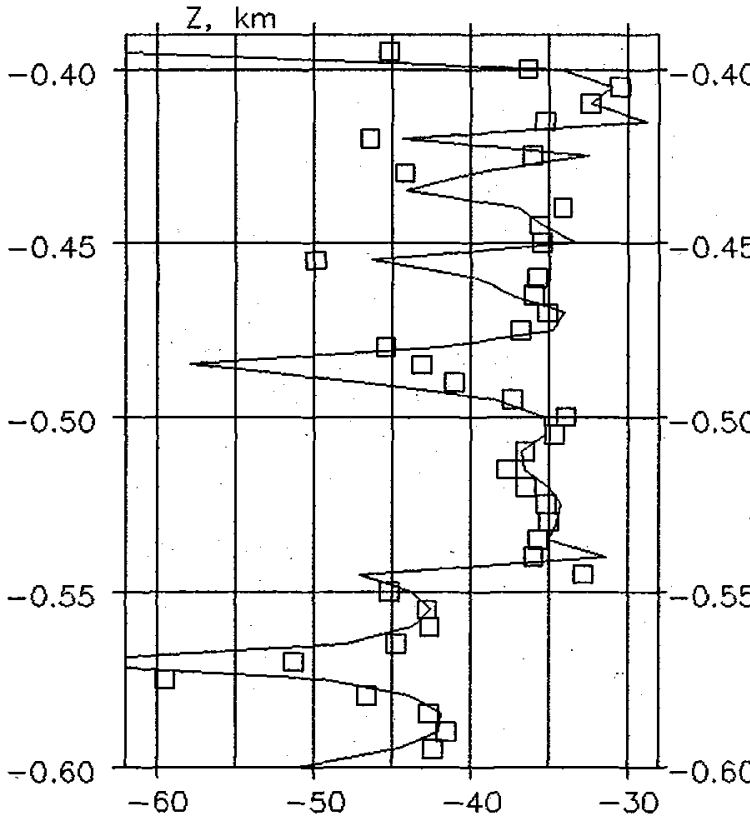

a)

I, d

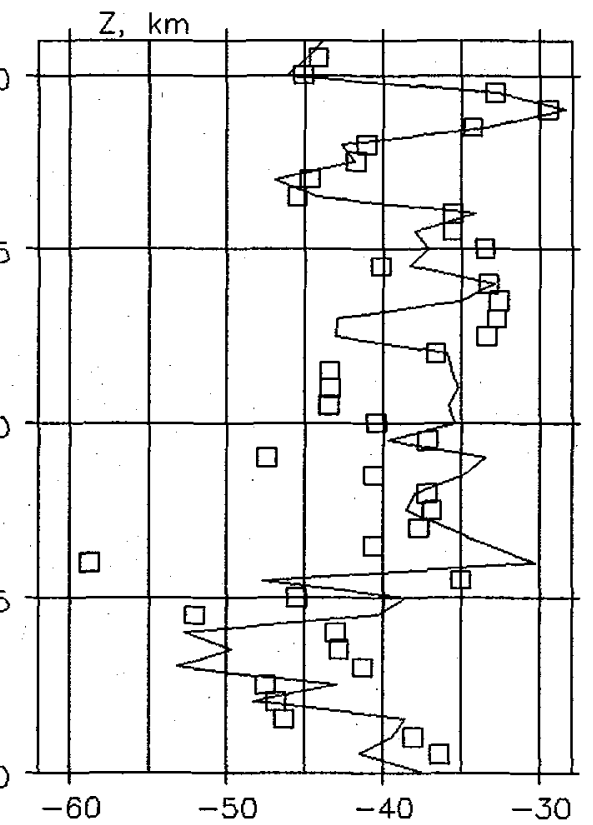

b)

1, dB

Fig.2. Depth dependence of transmission loss obtained via exact formulas of ray acoustic and within ESS approximation. (a) $\mathrm{f}=1 \mathrm{kHz}$. (b) $f=5 \mathrm{kHz}$. 
$c$ and $u$ are just the same as on Fig.1a. Source and receiver are both at depth $z_{s}=680$ $m$. The problem consists in determining $c(z)$ and $u(z)$ above this horizon.

Input data (i.e. times of flight of all eigenrays) were calculated for sourcereceiver separations $\mathrm{x}=\mathrm{iX} \mathrm{max}_{\max } / \mathrm{N}$, where $\mathrm{X}_{\max }=15 \mathrm{~km}$ is the distance from source to the boundary of shadow zone, $i=1,2, \ldots, N$ and $N$ is the number of points of measurements. Light circles, stars and squares stand for the difference between estimated and original profiles with $N=7, N=15$ and $N=20$ respectively. It turns out, that satisfactory accuracy of the inversion is presented even when the travel times between only 7 points are given. In practice, it is expedient to use the nonperturbative methods of inversion to obtain some initial estimate, of $c$ and $\vec{u}$ vertical dependence, which can be refined, including determining $c$ and $\vec{u}$ variability in horizontal plane, via standard linear inversion.

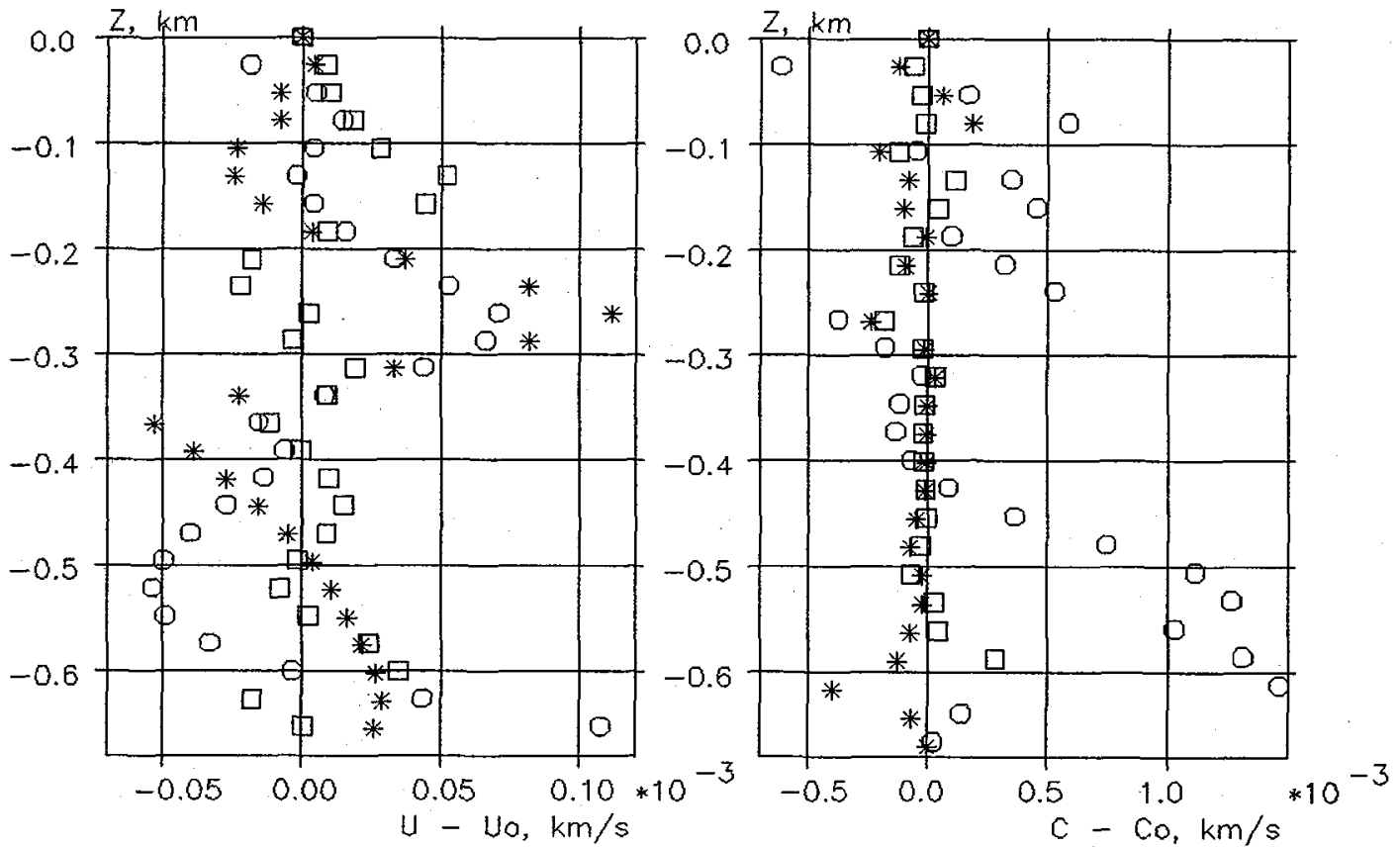

a)

b)

Fig. 3. Influence of number of rays used on accuracy of inversion of $u(z)(a)$ and $c(z)$ (b).

[1] MUNK, W.H., WUNSCH, C, Phil. Trans. Roy. Soc. London A307 (1982) 439

[2] HOWE, B.M, WORCESTER, P.F, SPINDEL, R.C, J. Geophys. Res. 92 (1987) 3795

[3] KO,D.S, DEFERRARI, H.A, MALANOTTE-RIZZOLI, P, J. Geophys. Res. 94 (1989) 6197

[4] GODIN, O.A, MIKHIN, D. YU, MOLCHANOV, S.YA, Izv. Acad. Sci. USSR Atmos. Ocean Phys. In press

[5] BERESUTSKI I, A.V, MAXIMOV,S.E., SKLYAROV,V.E, GORDON,R.L, J. Atmos. Ocean. Technol. In press

[6] ROBERTSON, J.S, SIEGMANN, W. L, JACOBSON, M. J, J. Acoust. Soc. Amer. 77 (1985) 1768

[7] RASMUSSEN, K.B, J. Sound Vibr. 104 (1986) 321

[8] BERAN, M.J, WHITMAN, A.M, J. Acoust. Soc. Amer. 81 (1987) 647

[9] NIJS, L, WAPENAAR, C.P.A, J. Acoust. Soc. Amer. 87 (1990) 1987

[10] GODIN, O.A, MIKHIN,D.YU., MOLCHANOV,S.YA, IzV. Acad. Sci. USSR Atmos. Ocean Phys. 27 (1991) 139 